\title{
WESTERBORK OBSERVATIONS OF THE GALACTIC SUPERNOVA REMNANTS IC 443 AND TYCHO*
}

\author{
R. M. DUIN, R. G. STROM, and H. VAN DER LAAN
}

Sterrewacht, Leiden, The Netherlands

\begin{abstract}
Continuum observations of IC 443 were made at $21 \mathrm{~cm}$ with a resolution of $23^{\prime \prime} \times 59^{\prime \prime}$. The appearance in the radio continuum is very similar to the optical appearance. Since the radio emission is nonthermal, this requires a mechanism that can enhance the synchrotron volume emissivity within thermal filaments, which emit strong optical radiation. We suggest compression of gas and magnetic fields in a shock.

Tycho's remnant has been observed both at $21 \mathrm{~cm}$ (resolution $24^{\prime \prime} \times 27^{\prime \prime}$ ) and at $6 \mathrm{~cm}$ (resolution $7^{\prime \prime} \times 8^{\prime \prime}$ ). Most of the fine scale structure is resolved. The similarity at the two wavelengths indicates a remarkable constancy in the spectral index of features down to a scale of $20^{\prime \prime}$.
\end{abstract}

\section{Introduction}

High resolution studies of extended radio sources require an aperture synthesis instrument of great sensitivity. The Westerbork Synthesis Radio Telescope (WSRT), with its twelve $25-\mathrm{m}$ paraboloids, admirably fulfills this requirement. Here we report on observations of two supernova remnants, IC 443 and 3C 10 (Tycho's remnant), made with sufficient resolution that about 5000 synthesized beams are required to fully scan each object.

\section{Observations of IC 443}

IC 443 is the remnant of a probable type II supernova of several tens of thousands of years ago. In the bright NE part of the remnant, filaments aligned in the tangential direction are readily apparent on the Palomar Sky Survey print (Figure 1). Temperatures of about $10^{4} \mathrm{~K}$ and densities on the order of $400 \mathrm{~cm}^{-3}$ are indicated for these filaments (Parker, 1964). To the west and southwest the filaments are weaker and define a more irregular morphology. It is likely that in these regions the interstellar medium is more tenuous than to the northeast, where $\mathrm{H}$ I observations suggest $n_{\mathrm{H}}=10 \mathrm{~cm}^{-3}$ for the interstellar density (Locke et al., 1964).

A short period $(P=0.34 \mathrm{~s})$ pulsar, PSR $0611+22$, has been discovered just to the west of the remnant (Davies et al., 1972). The period and slowing down rate define a time, $P / \dot{P}=190000$ yr, which by analogy with the Crab nebula pulsar suggests an age of $70000 \mathrm{yr}$. If a Sedov solution is applicable to the present shock front in IC 443, the average expansion rate should be 2.5 times the present speed of $65 \mathrm{~km} \mathrm{~s}^{-1}$ (Lozinskaya, 1969). Combined with the pulsar age, this would place the remnant at $2.5 \mathrm{kpc}$ rather than the much quoted distance of $1.5 \mathrm{kpc}$.

Continuum observations of IC 443 were made with the WSRT at a wavelength of $21 \mathrm{~cm}$. The high resolution $\left(23^{\prime \prime} \times 59^{\prime \prime}\right)$ map in Figure 2 reveals an overwhelming amount of fine scale structure, especially in the northeastern sector. To the southwest,

* Presented by H. J. Habing. 


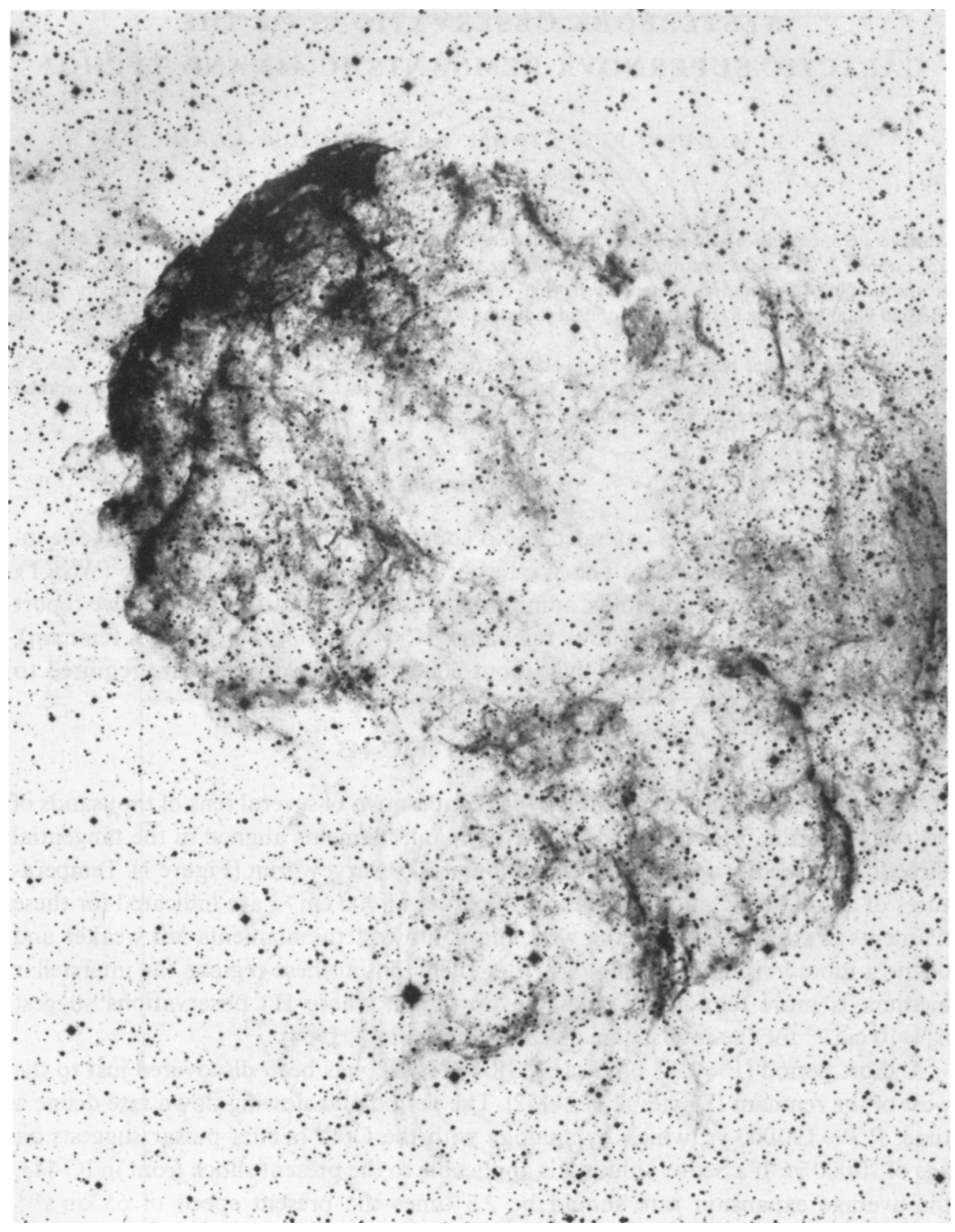

Fig. 1. A copy of the red Palomar Sky Survey plate of IC 443. The main contribution to the optical picture comes from line radiation $(\mathbf{H} \alpha)$. (Copyright National Geographic Society - Palomar Observatory.) 


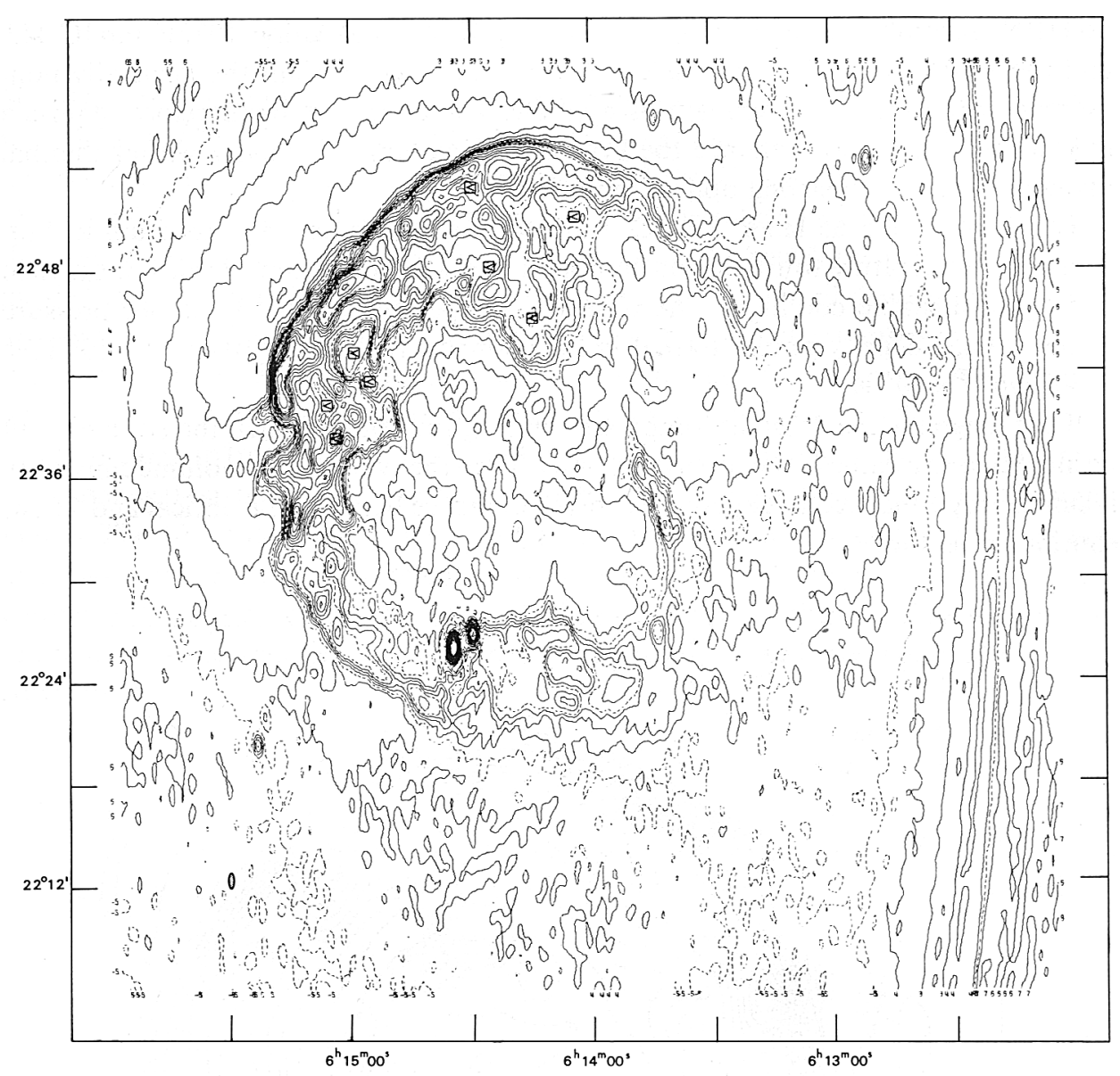

Fig. 2. Contour map of the total intensity of IC 443 at $21-\mathrm{cm}$ continuum. The outermost dashed contour is the apparent zero level. From that level the contour intervals between pairs of dashed contours are 1.1, 2.2 , and $4.4 \mathrm{~K}$, respectively. The symbol ' $<$ ' enclosed in a square indicates a local minimum. The shaded ellipse in the SE corner represents the half-power beam.

the signal to noise ratio is considerably degraded by primary beam attenuation (the field center is at $R A=6^{\mathrm{h}} 14^{\mathrm{m}} 36^{\mathrm{s}}$, Dec $\left.=22^{\circ} 42^{\prime} 6^{\prime \prime}\right)$. It should be noted that because of the shortest baseline $(36 \mathrm{~m})$ a considerable amount of large scale structure is absent from the map.

Comparing the optical and radio morphology, a great similarity is apparent. This spatial coincidence has been noted by Hill (1971) but our smaller beam confirms it in still greater detail. Such a close correlation would be expected in an $\mathrm{H}$ II region, for example, where the (optical) line emission and (radio) free-free emission originate in the same material. It is surprising, however, in a supernova remnant whose radio emission almost certainly originates in a low density gas of relativistic particles radiating in a magnetic field. It is unlikely that the radio emission is thermal in origin: 
no $\mathrm{H} 109 \alpha$ recombination line has been detected (Dickel and Milne, 1972) from IC 443, and the spectrum of the northeastern part is even steeper than the decidedly nonthermal spectrum of the remnant as a whole. The observations therefore require a mechanism which can enhance the synchrotron volume emissivity within thermal filaments which emit strong optical radiation.

The passage of a $65 \mathrm{~km} \mathrm{~s}^{-1}$ shock front through the interstellar medium will increase its temperature to $10^{5} \mathrm{~K}$ and compress it by a factor of four. If this hot gas then undergoes unstable cooling to the observed temperature of $10^{4} \mathrm{~K}$, under pressure equilibrium, a density similar to that in the filaments results: $4 \times 1 \mathrm{~cm}^{-3} \times 10^{5} \mathrm{~K}=$ $=400 \mathrm{~cm}^{-3} \times 10^{4} \mathrm{~K}$.

If the magnetic field also is compressed during the cooling, then the strength will be increased to a value of the order of $10^{-4} \mathrm{G}$. Then the synchrotron volume emissivity is sufficiently enhanced to explain the correspondence between the optical and $21 \mathrm{~cm}$ continuum features.

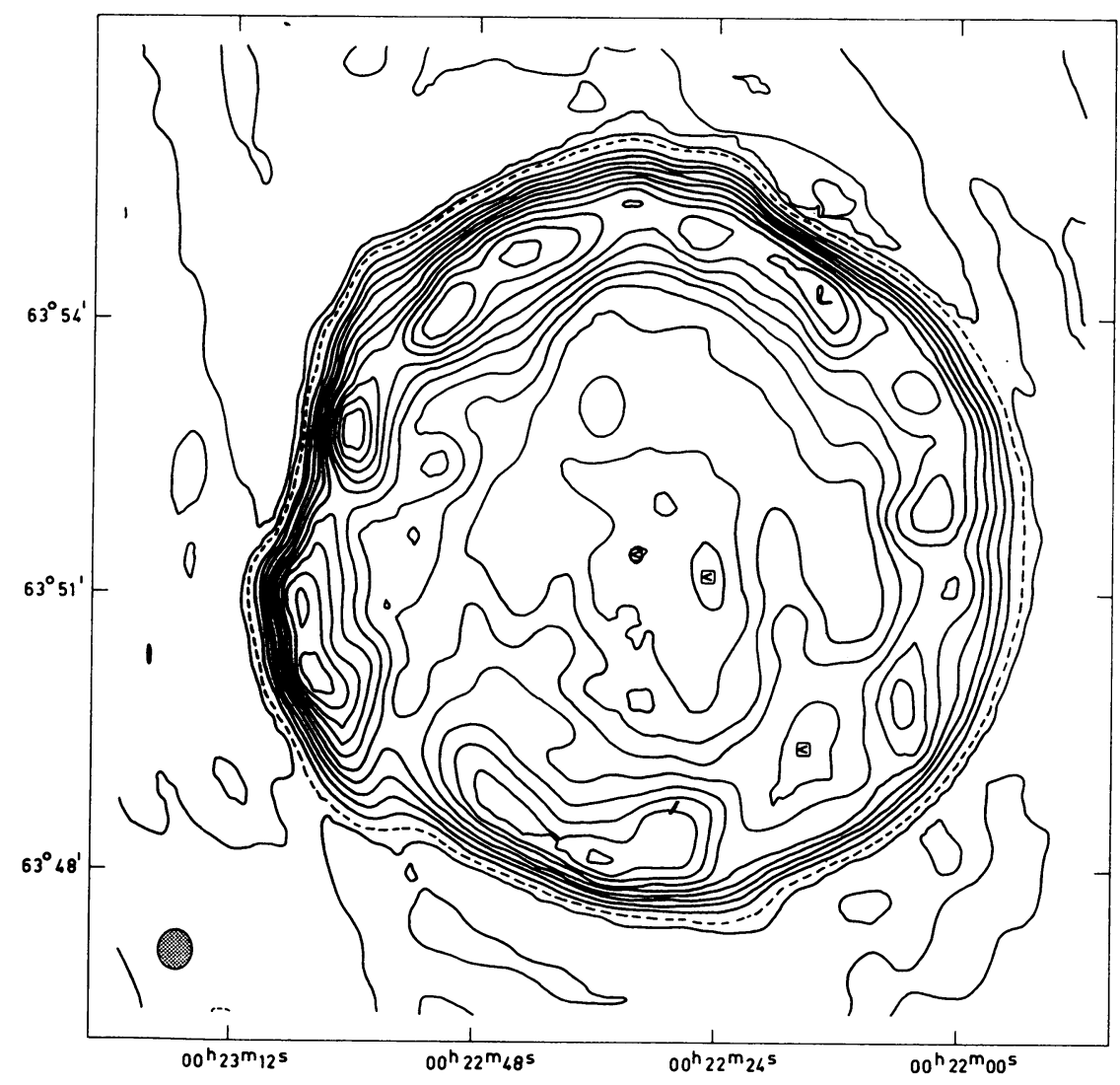

Fig. 3. Contours of total intensity for Tycho's remnant at $21-\mathrm{cm}$ continuum. Above the map zero level, which is dashed, the contour interval is $27.5 \mathrm{~K}$. The symbol ' $<$ ' enclosed in a square indicates a local minimum. The shaded ellipse represents the synthesized half-power beam. 


\section{Observations of 3C 10 (Tycho's Remnant)}

3C 10 has now been observed at two wavelengths with the WSRT. A $21-\mathrm{cm}$ continuum map (with a $24^{\prime \prime} \times 27^{\prime \prime}$ synthesized beam) of the total intensity and polarized emission has been discussed by Strom and Duin (1973). The total intensity map is reproduced in Figure 3. Among its features are the remarkable circularity of the ring of emission, the steep outer gradients and the fine scale structure. Although the peaks of emission inside the main ring (many of which are not apparent because of the contour interval) are generally weaker than those superimposed on top of it, the number in each group is about the same. Special attention is drawn to the interior ridge at $R A=0^{\mathrm{h}} 22^{\mathrm{m}} 19^{\mathrm{s}}$ which extends northwards from the main ring to at least $\mathrm{Dec}=63^{\circ} 52^{\prime}$.

The 6-cm map (resolution $7^{\prime \prime} \times 8^{\prime \prime}$ ) is shown in Figure 4, and it is apparent that most of the fine scale structure is resolved, although the outer edge still is unresolved in some places. The aforementioned interior ridge now appears as a string of emission peaks, and many of the other components observed at $21 \mathrm{~cm}$ split up into numerous subcomponents. In some cases it is clear from the 6-cm map that the more inward position of the outermost peaks in the $21-\mathrm{cm}$ map is the result of the larger beam at the latter wavelength.

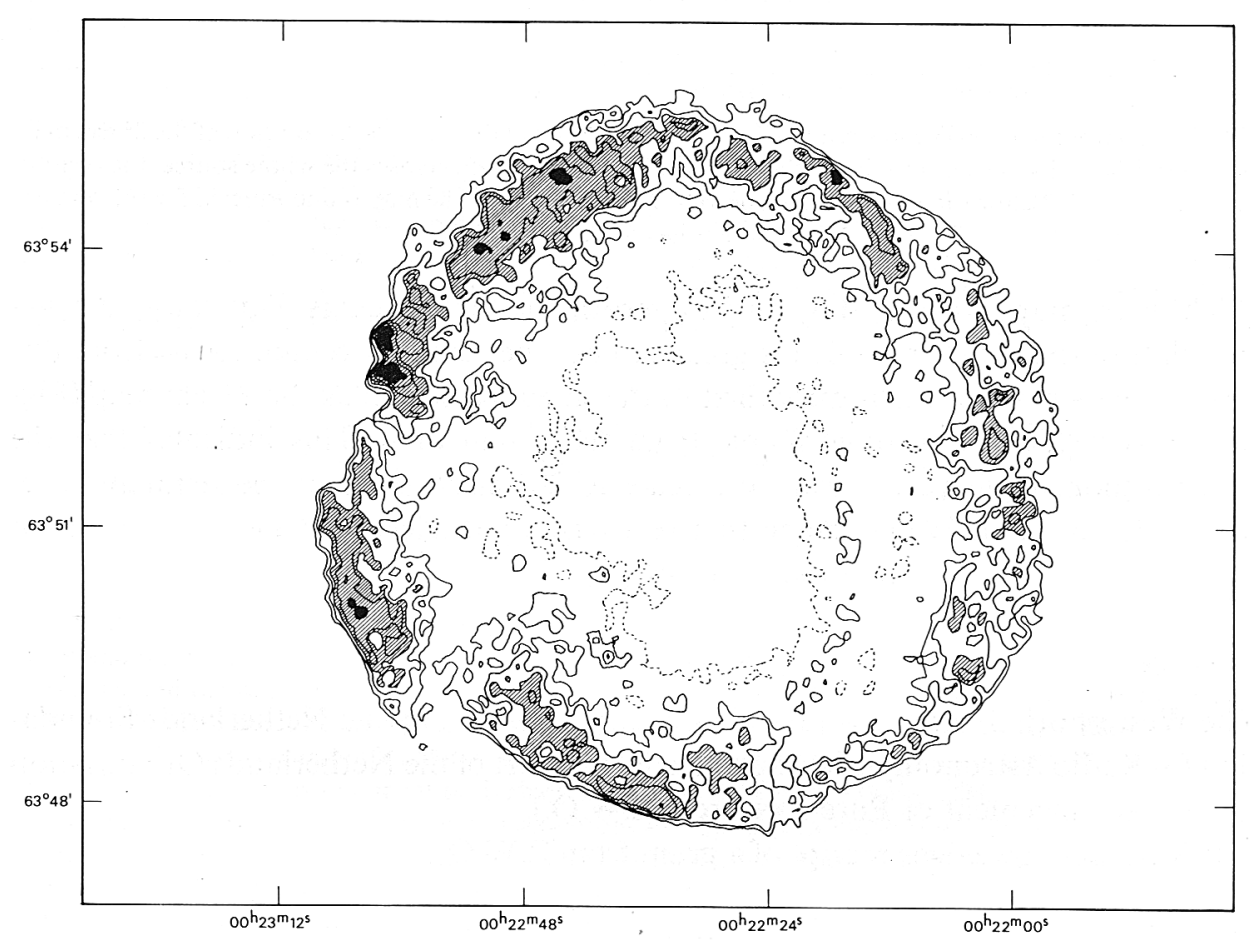

Fig. 4. Contour map of Tycho's remnant at $6 \mathrm{~cm}$. The resolution is $7 " \times 8^{\prime \prime}$. For the sake of clarity only some positive contours are shown. Regions above $8.25 \mathrm{~K}$ are shown black, while everything above $4.13 \mathrm{~K}$ is shaded. The outermost contour is $1.38 \mathrm{~K}$ above the apparent zero level (see text). The map is uncorrected for the primary beam, which has a HPBW of $10.6^{\prime}$. 


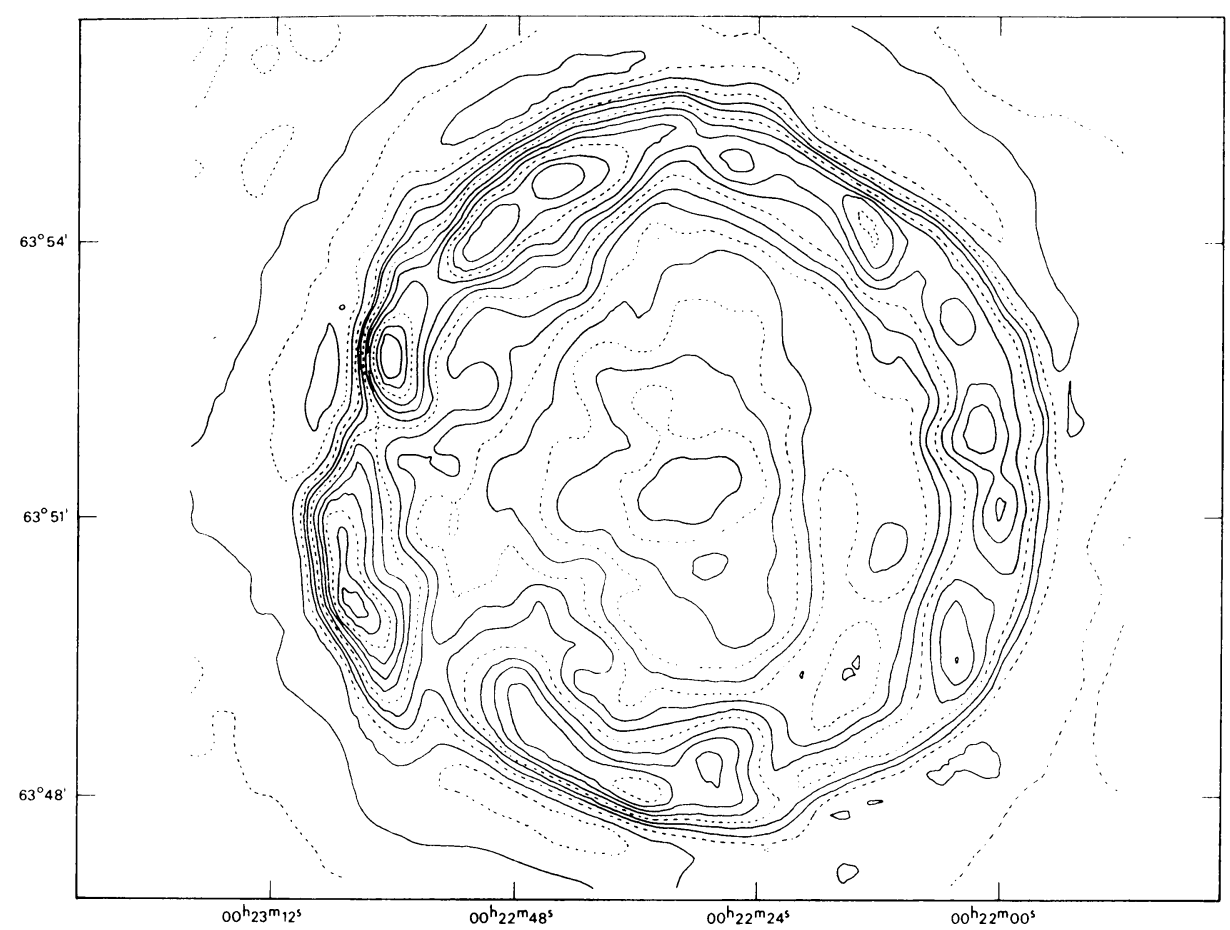

Fig. 5. Contour map of Tycho's remnant at $6 \mathrm{~cm}$, convolved to the $24^{\prime \prime} \times 27^{\prime \prime}$ resolution of the $21-\mathrm{cm}$ map. Above the dashed apparent zero level, the first dashed contour that encloses the whole source, the contour interval is $0.67 \mathrm{~K}$. Below the zero level the contour interval is $0.9 \mathrm{~K}$. The map is uncorrected for the primary beam, which has a HPBW of 10.6'.

The 6-cm map is distorted by missing zeroth and first spacings. This causes a negative shift of the contours which is greatest near the remnant center. Apart from this effect, the 6-cm map, when smoothed to the same resolution as the 21-cm map (Figure 5), is virtually indistinguishable from the $21-\mathrm{cm}$ map. This indicates that the spectral index of features down to a scale of $20^{\prime \prime}$ in all parts of the remnant is remarkably constant. We conclude that relativistic particles throughout the remnant must have identical acceleration histories.

\section{Acknowledgements}

The Westerbork Synthesis Radio Telescope is operated by the Netherlands Foundation for Radio Astronomy with the financial support of the Netherlands Organisation for the Advancement of Pure Research (Z.W.O.).

R.M.D. acknowledges receipt of a grant from Z.W.O.

\section{References}

Davies, J. G., Lyne, A. G., and Seiradakis, J. D. : 1972, Nature 240, 229.

Dickel, J. R. and Milne, D. K.: 1972, Australian J. Phys. 25, 539.

Hill, I. E.: 1972, Monthly Notices Roy. Astron. Soc. 157, 419. 
Locke, J. L., Galt, J. A., and Costain, C. H.: 1964, Astrophys. J. 139, 1071.

Lozinskaya, T. A.: 1969, Soviet Astron. 13, 192.

Parker, R. A. R.: 1964, Astrophys. J. 139, 493.

Strom, R. G. and Duin, R. M.: 1973, Astron. Astrophys. 25, 351.

\author{
R. M. Duin, R. G. Strom, and H. van der Laan \\ Sterrewacht, Huygens Laboratorium, Wassenaarseweg 78 , \\ Leiden 2405, The Netherlands
}

\title{
DISCUSSION
}

Menon: I would like to know whether that steep gradient is resolved or still unresolved at 6-cm resolution? Habing: It is unresolved.

J. R. Dickel: Hirabayashi's map agrees very closely with the 2-cm map (with $2^{\prime}$ beam) by Mayer and Hollinger (Astrophys. J. 151, (1968), 53). Our 11 -cm map of Tycho (Hermann and Dickel: 1973, Astron. J. No. 9) shows the same cell structure in the polarization as Strom and Duin find at $21 \mathrm{~cm}$, supporting their conclusion of a thin-screen Faraday rotation in front of the source. 3C 391 is so poorly resolved by us at $8.8 \mathrm{GHz}$ that I would be leery of attaching much significance to any spectrai features found by Slee and Dulk based on that map. In CTB 37A the amount of significant fall-off in the spectrum at low frequencies depends critically upon the point at $408 \mathrm{MHz}$ by Kesteven, which has recently been revised downward by D. Clarke. If real, the very uniform absorption mentioned by Slee must have a very abrupt boundary because CTB 37B only $20^{\prime}$ to the north (but presumably more distant based upon the $\Sigma-D$ relation) does not show significant absorption. A uniform cloud which falls abruptly at the edge of a source must lie very close to the source itself.

Shakeshaft: Although we have seen the remarkable similarity of maps of several supernova remnants over very wide ranges of frequency, there is good evidence in IC 443 of changes with frequency, the bright rim at the north-east having a spectrum steeper than that of the rest of the source. (Hill, I. E.: 1973, Monthly Notices Roy. Astron. Soc. 164, 398). Further, there is a good opportunity to obtain spectral information at low frequencies from observations of the lunar occultations occurring during the next year.

J. R. Dickel: But for integrated spectrum be careful of the point sources on the south (as Duin presented to Lecce symposium on supernovae, May 1973) which might be unrelated and of different spectral index.

Kerr: Kundu and Velusamy, at University of Maryland, have done rather detailed polarization observations on several dozen northern sources from Green Bank at 3 and $11 \mathrm{~cm}$. Their work gives results which support Dickel's main conclusions.

Mills: Some of Slee's supernova remnants are very, very small. Could these be extragalactic objects? At very small angular size, it is difficult to decide whether it is a supernova remnant or an extragalactic object. Has there been any independent evidence, say on whether the spectrum is very flat or something like that?

Slee: In most of these cases the spectral index is 0.5 or 0.6 or less. I don't think any polarization measurements have been made on many of them.

Oort: I understood from Dickel's communication that the 'cell' structure he found in the polarization measurements in the Vela SNR could not be due to Faraday rotation. This is interesting in connection with the much more pronounced cellular structure found by Strom and Duin at Leiden in Tycho's SNR at $21 \mathrm{~cm}$ and $6 \mathrm{~cm}$. This structure was suggested by the authors and van der Laan to be due to structure in a screen around the radio SNR. Dickel's observations in the Vela object may indicate that the thin shield might not be the correct explanation, but that the structure would be inherent to the shell itself.

Dickel: The Vela structure looks more like a continuous whorl rather than the small discrete cells we see in Tycho. See also my comment on Habing's report of Strom and Duin's work.

Parijskij: I would like to report that observations by Soboleva at Pulkovo at $2 \mathrm{~cm}$ with $40^{\prime \prime}$ resolution of the Crab nebula give evidence of the existence of a cavity around the Crab pulsar but of much larger size than we may expect from low frequency radiation pressure.

Jenkins: In connection with our guest investigator program on Copernicus, we have observed through the Vela supernova several stars. They have interstellar line components at about $-80 \mathrm{~km} \mathrm{~s}^{-1}$ and -150 $\mathrm{km} \mathrm{s}^{-1}$; I don't think we'll see much difference in the composition of the gas. It's probably mostly interstellar material swept up. We can get a measure of the ionization balance for the gas in this region. We have not yet had a chance to analyze the results, but a quick look seems to show that it is a higher than usual level of ionization. 